\title{
Ultrasonic Studies of Copper Soap-urea Complexes Derived from Mustard and Soybean Oils
}

\author{
Arun Kumar Sharma, ${ }^{1 *}$ Meenakshi Saxena ${ }^{2}$ and Rashmi Sharma ${ }^{3}$ \\ ${ }^{1}$ Department of Chemistry, Govt. P.G. College Jhalawar, 326001 Rajasthan, India \\ ${ }^{2}$ Department of Chemistry, S.D. Govt. College Beawar, 305901, Rajasthan, India \\ ${ }^{3}$ Department of Chemistry, S.P.C. Govt. College Ajmer, 305001 Rajasthan, India \\ *Corresponding author: sharmaarun423@gmail.com
}

Published online: 25 November 2018

To cite this article: Sharma, A. K., Saxena, M. \& Sharma, R. (2018). Ultrasonic studies of copper soap-urea complexes derived from mustard and soybean oils. J. Phys. Sci., 29(3), 67-82, https://doi.org/10.21315/jps2018.29.3.6

To link to this article: https://doi.org/10.21315/jps2018.29.3.6

\begin{abstract}
Ultrasonic velocities and densities have been measured in ternary mixtures containing copper soap-urea complexes derived from mustard and soybean oils in 20\% and 40\% methanol-benzene mixture to assess the effect of polarity of solvent on the micellar features of surfactant. The studies suggest that predomination of benzene (higher percentage of non-polar solvent) plays a significant role in compacting the molecular organisation of the micelles. From these values, the ultrasonic velocity $(u)$, the specific acoustic impedance $(Z)$, adiabatic compressibility $(\beta)$, intermolecular free length $\left(L_{f}\right)$, apparent molar compressibility $\left(f_{k}\right)$, molar sound velocity $(R)$ and primary solvation number $\left(S_{n}\right)$ have been calculated. The data clearly indicate that the values of $u, Z, S_{n}$ and $R$ increase whereas the values $\beta$ and $L_{f}$ decrease consistently with increase in the soap complex concentration. The critical micelle concentration (CMC) has also been determined and it has been found that CMC is dependent on the composition of the solvent mixture and composition of the edible oil. The results have been analysed in terms of Masson's equation. The results have been explained on the basis of intermolecular interactions between solute and solvent molecules and effect of polarity and concentration were accessed on various acoustic parameters.
\end{abstract}

Keywords: Copper soap-urea complex, methanol, benzene, acoustic parameters, intermolecular interactions 


\section{INTRODUCTION}

Study of propagation of ultrasonic waves and their absorption forms one of the most important methods of investigation of properties of matter in all the three states. It is well known that study of absorption of ultrasonic waves in a medium provides important information about various inter and intra-molecular processes such as relaxation of the medium or the existence of isomeric states or the exchange of energy between various molecular degrees of freedom..$^{1-6}$ Derived parameters from ultrasonic velocity measurements provide qualitative information regarding the nature and strength of molecular interactions in liquid mixtures. ${ }^{7,8}$ This has been studied for various binary and ternary mixtures with respect to variation in concentration of the liquids and temperatures. ${ }^{9,10}$ Ultrasonic velocity measurements have been extensively used to study the molecular interactions in electrolyte solutions and to get significant information on the arrangement of molecules in aqueous electrolyte solutions. ${ }^{11-15}$ In the present investigation, we have studied various thermo-acoustical parameters, such as specific acoustic impedance $(\mathrm{Z})$, adiabatic compressibility $(\beta)$, intermolecular free length $\left(\mathrm{L}_{\mathrm{f}}\right)$, apparent molar compressibility, $\left(f_{k}\right)$, molar sound velocity $(R)$ and primary solvation number $\left(\mathrm{S}_{\mathrm{n}}\right)$ to examine the ion-solvent and ion-ion interactions in the aqueous solutions of soap urea complexes made by mustard and soybean oils which have wide applications in pharmaceutical, medicinal, agricultural, environmental, textile and leather industries. ${ }^{16-20}$

The present work deals with the determination of ultrasonic velocity measurements which have been used to obtain information regarding various acoustic parameters and solute-solute and solute-solvent interactions of solutions of $\mathrm{Cu}$ (II) soap urea complex of edible oil of mustard and soybean in pure non-polar solvents, polar and non-polar solvent mixtures (benzene and its varying compositions with methanol) to assess the effect of polarity of solvent molecules on the micellar nature of solute molecule. The studies have also been done to understand the nature of the solution and observed that the increase in the internal pressure and in lowering the compressibility of the solution. Benzene as a solvent has been used due to maximum solubility of soaps is this non-aqueous solvent.

\section{EXPERIMENTAL}

All the chemicals used were of lab reagent/analytical reagent (LR/AR) grade. Copper soap was prepared by refluxing the mustard and soybean oils (mustard oil extracted from seeds and purified, and soybean oil available in market with reputed brand) with ethyl alcohol and $2 \mathrm{~N} \mathrm{KOH}$ solutions for 3-4 h (direct metathesis). The neutralisation of excess $\mathrm{KOH}$ present was done by slow addition 
of $0.5 \mathrm{~N} \mathrm{HCl}$. Saturated solution of copper sulphate was then added to it, for conversion of neutralised potassium soap into their corresponding copper soaps. Copper soap so obtained was then washed with warm water and $10 \%$ alcohol at $50^{\circ} \mathrm{C}$ and recrystallised using hot benzene. Molecular weights of copper soaps were determined from saponification values. ${ }^{21}$ The copper soap complexes are abbreviated as copper-mustard urea (CMU) and copper-soybean urea (CSoU). Fatty acid composition of oils used for synthesis of $\mathrm{Cu}$ (II) soaps and their analytical and physical data are summarised in Tables 1 and 2.

Table 1: Fatty acid composition of oils used for copper urea complexes synthesis.

\begin{tabular}{lcccccc}
\hline \multirow{2}{*}{ Name of oil } & \multicolumn{6}{c}{$\%$ Fatty acids } \\
\cline { 2 - 7 } & $16: 0$ & $18: 0$ & $18: 1$ & $18: 2$ & $18: 3$ & Other acids \\
\hline Mustard oil & 2 & 1 & 25 & 18 & 10 & $\mathrm{C}_{20}-\mathrm{C}_{22} 41 \%$ \\
Soybean oil & 12 & 4 & 24 & 51 & 9 & - \\
\hline
\end{tabular}

Table 2: Analytical and physical data of copper urea complexes derived from mustard and soybean oils.

\begin{tabular}{lccccc}
\hline \multirow{2}{*}{ Compound } & \multirow{2}{*}{ Colour } & \multirow{2}{*}{ Melting point $\left({ }^{\circ} \mathrm{C}\right)$} & \multicolumn{2}{c}{ Metal \% } & \multirow{2}{*}{ Mol.wt. } \\
\cline { 4 - 5 } & & 72 & Found & Calculated & \\
\hline CMU & Dark green & 100 & 9.46 & 8.35 & 759.72 \\
CSoU & Dark green & 10.31 & 9.10 & 697.17 \\
\hline
\end{tabular}

The ultrasonic velocity was determined with the help of a single crystal ultrasonic interferometer model M-82 (Mittal Enterprises, New Delhi) working at a fixed frequency of $2 \mathrm{MHz}$. The temperature of water was maintained at $30^{\circ} \mathrm{C} \pm 0.1^{\circ} \mathrm{C}$ by a thermostat through the jacket of the cell before the measurement was actually made. The measured velocities have an uncertainty of $0.5 \mathrm{~m} \mathrm{~s}^{-1}$. Further, the operative part of the measurement involves the slow movement of micrometer till the anode current meter shows a maximum. A number of maxima readings of anode current are passed on and their number $(n)$ is counted. The total distance $(d)$ thus moved by the micrometer gives the value of wavelength $(\lambda)$ with the help of the following relation:

$$
d=n \times \lambda
$$

Once the wavelength is known, the velocity $(\mathrm{u})$ in the liquid can be calculated with the help of the following relation:

$$
\mathrm{u}=\lambda \times f
$$

where $f$ is the frequency of instrument used, i.e., $2 \mathrm{MHz}$. 


\section{ACOUSTIC PARAMETERS}

The values of $\mathrm{Z}, \beta, \mathrm{L}_{\mathrm{f}}, \phi_{\mathrm{k}}, \mathrm{R}$ and $\mathrm{S}_{\mathrm{n}}$ are calculated by using the following relationships: ${ }^{22}$

$$
\begin{aligned}
& \mathrm{Z}=\mathrm{u} \rho \\
& \beta=\frac{1}{\mathrm{u}^{2} \rho} \\
& \mathrm{L}_{\mathrm{f}}=\mathrm{K} \sqrt{\beta} \\
& \phi_{\mathrm{k}}=\left[1000 \cdot \frac{\beta \rho_{0}-\beta_{0} \rho}{\mathrm{c} \rho_{0}}\right]+\frac{\beta \mathrm{M}}{\rho_{0}} \\
& \mathrm{R}=\mathrm{M} \cdot(\mathrm{u}) \frac{1}{3} \rho_{0} \\
& \mathrm{M}=\mathrm{X}_{1} \mathrm{M}_{1}+\mathrm{X}_{2} \mathrm{M}_{2}+\mathrm{X}_{3} \mathrm{M}_{3} \\
& \mathrm{~S}_{\mathrm{n}}=\left[\frac{\mathrm{n}^{0}}{\mathrm{n}}\right] \cdot\left[1-\frac{\mathrm{V} \beta}{\mathrm{n}^{0} \mathrm{~V}^{0} \beta^{0}}\right]
\end{aligned}
$$

where $\mathrm{b}_{0}, \beta, \mathrm{r}_{0}, \rho, \mathrm{n}_{0}, \mathrm{n}, \mathrm{V}_{0}$ and $\mathrm{V}$ are adiabatic compressibility, density, numbers of moles and molar volume of solvent and solute, respectively. The expression for molar volume of solvent $V_{0}$ and soap solution $V$ may be written as:

$$
\begin{aligned}
& \mathrm{V}=\mathrm{X}_{1} \mathrm{M}_{1}+\mathrm{X}_{2} \mathrm{M}_{2}+\mathrm{X}_{3} \mathrm{M}_{3} / \rho \\
& \mathrm{V}_{0}=\mathrm{X}_{1} \mathrm{M}_{1}+\mathrm{X}_{2} \mathrm{M}_{2}+\mathrm{X}_{3} \mathrm{M}_{3} / \rho_{0}
\end{aligned}
$$

In Equations 6, 8 and 9, $\mathrm{M}$ represent the molecular weight and $\mathrm{X}$ the mole fraction of the component. Here the subscripts 1,2 and 3 refer to soap, methanol and benzene, respectively.

\section{RESULTS AND DISCUSSION}

\subsection{Copper Soap-urea Complex Derived from Mustard and Soybean Oils in 20\% Methanol-benzene Mixture}

\subsubsection{Acoustic parameters}

After perusal of the observed data, it may be mentioned that the acoustic parameters and equations related to ultrasonic investigations are the same as has been enumerated for the previous soaps. The values of ultrasonic velocity and allied parameters for these complexes in methanol-benzene mixture are given in Tables 3 and 4 . 
A perusal of the data suggests that the values of $u, Z, \beta, \phi_{k}, R$ and $S_{n}$ increase with increasing complex concentration whereas $\beta$ and $\mathrm{L}_{\mathrm{f}}$ decrease with the increase in the concentration. $\mathrm{Z}$ has a greater significance and as a characteristic property of the medium than either density or ultrasonic velocity individually. ${ }^{23}$

Table 3: Ultrasonic velocity and other acoustic parameter of copper urea complex derived from mustard oil in $20 \%$ methanol-benzene mixture $\left(\mathrm{CMU}_{20}\right)$.

\begin{tabular}{ccccccccc}
\hline $\begin{array}{c}\mathrm{c} \\
\left(\mathrm{mol} \mathrm{l}^{-1}\right)\end{array}$ & $\begin{array}{c}\rho \\
\left(\mathrm{g} \mathrm{dm}^{-3}\right)\end{array}$ & $\begin{array}{c}\mathrm{u} \\
\left(\mathrm{m} \mathrm{s}^{-1}\right)\end{array}$ & $\begin{array}{c}\mathrm{Z} \times 10^{-5} \\
\left(\mathrm{~g} \mathrm{~cm}^{-2} \mathrm{~s}^{-1}\right)\end{array}$ & $\begin{array}{c}\mathrm{b}_{\mathrm{ad}} \times 10^{11} \\
\left(\mathrm{~cm}^{2} \mathrm{dyn}^{-1}\right)\end{array}$ & $\mathrm{L}_{\mathrm{f}} \times 10^{10}\left(\mathrm{~A}^{0}\right)$ & $-\phi_{\mathrm{k}} \times 10^{8}$ & $\mathrm{R} \times 10^{-2}$ & $\mathrm{~S}_{\mathrm{n}} \times 10^{-3}$ \\
\hline 0.0003 & 0.9548 & 1538.5 & 1.4689 & 4.4250 & 1.3171 & 4220.7 & 34.700 & -57.757 \\
0.0004 & 0.9556 & 1544.4 & 1.4758 & 4.3874 & 1.3115 & 3270.5 & 34.719 & -42.598 \\
0.0006 & 0.9565 & 1545.6 & 1.4784 & 4.3765 & 1.3099 & 2205.9 & 34.701 & -28.232 \\
0.0008 & 0.9574 & 1548.6 & 1.4826 & 4.3555 & 1.3067 & 1686.2 & 34.696 & -20.960 \\
0.0010 & 0.9559 & 1563.1 & 1.4942 & 4.2816 & 1.2956 & 1413.0 & 34.864 & -16.302 \\
0.0011 & 0.9564 & 1565.6 & 1.4973 & 4.2660 & 1.2932 & 1301.0 & 34.867 & -14.708 \\
0.0012 & 0.9569 & 1569.2 & 1.5016 & 4.2438 & 1.2899 & 1213.2 & 34.879 & -13.342 \\
0.0013 & 0.9574 & 1571.1 & 1.5042 & 4.2316 & 1.2880 & 1131.2 & 34.877 & -12.239 \\
0.0014 & 0.9579 & 1574.2 & 1.5079 & 4.2128 & 1.2851 & 1065.7 & 34.885 & -11.262 \\
0.0016 & 0.9586 & 1579.2 & 1.5138 & 4.1832 & 1.2806 & 953.0 & 34.902 & -9.714 \\
0.0018 & 0.9595 & 1584.2 & 1.5200 & 4.1530 & 1.2760 & 866.4 & 34.912 & -8.504 \\
0.0020 & 0.9605 & 1588.6 & 1.5258 & 4.1257 & 1.2718 & 795.9 & 34.913 & -7.546 \\
\hline
\end{tabular}

Table 4: Ultrasonic velocity and other acoustic parameter of copper urea complex derived from soybean oil in $20 \%$ methanol-benzene mixture $\left(\mathrm{CSoU}_{20}\right)$.

\begin{tabular}{ccccccccc}
\hline $\begin{array}{c}\mathrm{c} \\
\left(\mathrm{mol} \mathrm{l}^{-1}\right)\end{array}$ & $\begin{array}{c}\rho \\
\left(\mathrm{g} \mathrm{dm}^{-3}\right)\end{array}$ & $\begin{array}{c}\mathrm{u} \\
\left(\mathrm{m} \mathrm{s}^{-1}\right)\end{array}$ & $\begin{array}{c}\mathrm{Z} \times 10^{-5} \\
\left(\mathrm{~g} \mathrm{~cm}^{-2} \mathrm{~s}^{-1}\right)\end{array}$ & $\begin{array}{c}\mathrm{b}_{\mathrm{ad}} \times 10^{11} \\
\left(\mathrm{~cm}^{2} \mathrm{dyn}^{-1}\right)\end{array}$ & $\mathrm{L}_{\mathrm{f}} \times 10^{10}\left(\mathrm{~A}^{0}\right)$ & $-\phi_{\mathrm{k}} \times 10^{8}$ & $\mathrm{R} \times 10^{-2}$ & $\mathrm{~S}_{\mathrm{n} \times 10^{-3}}$ \\
\hline 0.0003 & 0.9554 & 1533.2 & 1.4648 & 4.4529 & 1.3213 & 4140.1 & 34.638 & -58.343 \\
0.0004 & 0.9562 & 1535.5 & 1.4683 & 4.4355 & 1.3187 & 3159.3 & 34.629 & -43.393 \\
0.0006 & 0.9572 & 1539.1 & 1.4732 & 4.4105 & 1.3150 & 2156.5 & 34.625 & -28.590 \\
0.0008 & 0.9584 & 1540.8 & 1.4767 & 4.3948 & 1.3126 & 1644.9 & 34.600 & -21.262 \\
0.0010 & 0.9592 & 1548.6 & 1.4854 & 4.3473 & 1.3055 & 1367.4 & 34.634 & -16.656 \\
0.0011 & 0.9598 & 1551.6 & 1.4892 & 4.3278 & 1.3026 & 1263.7 & 34.637 & -15.003 \\
0.0012 & 0.9604 & 1553.4 & 1.4919 & 4.3150 & 1.3006 & 1171.6 & 34.632 & -13.664 \\
0.0013 & 0.9585 & 1559.5 & 1.4947 & 4.2901 & 1.2969 & 1091.7 & 34.748 & -12.526 \\
0.0014 & 0.9590 & 1561.3 & 1.4973 & 4.2778 & 1.2950 & 1024.3 & 34.746 & -11.561 \\
0.0016 & 0.9598 & 1564.3 & 1.5014 & 4.2576 & 1.2919 & 911.4 & 34.745 & -10.014 \\
0.0018 & 0.9606 & 1567.4 & 1.5056 & 4.2374 & 1.2889 & 823.5 & 34.744 & -8.812 \\
0.0020 & 0.9615 & 1570.5 & 1.5100 & 4.2169 & 1.2858 & 753.7 & 34.739 & -7.847 \\
\hline
\end{tabular}

The values of $\mathrm{Z}$ follow the order: $\mathrm{CMU}>\mathrm{CSoU}$. 
This indicates that lyophobic interaction between complex and solvent molecules is higher in CSoU than those in CMU. It may be suggested that as $>\mathrm{C}=\mathrm{O}$ and $\mathrm{NH}_{2}$ groups of urea are capable of making intermolecular hydrogen bonding with other CSoU molecules, their interactions strength contribution is highest. For both complexes, the values of $\beta$ and $\mathrm{L}_{\mathrm{f}}$ decrease with increasing complex concentration which indicates that there are significant interactions between complex and solvent molecules. It is interesting to point out that the values of the parameters $\mathrm{u}, \mathrm{Z}, \beta$ and $\mathrm{L}_{\mathrm{f}}$ with respect to chain length of the complex follow the order:

$$
\begin{aligned}
& {[\mathrm{u}]: \mathrm{CMU}>\mathrm{CSoU}} \\
& {[\mathrm{Z}]: \mathrm{CMU}>\mathrm{CSoU}} \\
& {[\mathrm{b}]: \mathrm{CMU}<\mathrm{CSoU}} \\
& {\left[\mathrm{L}_{\mathrm{f}}\right]: \mathrm{CMU}<\mathrm{CSoU}}
\end{aligned}
$$

For both complexes, the $\mathrm{S}_{\mathrm{n}}$ increases with the increase in complex concentration signifying the aggregation of solvent molecules around the solute moiety. Negative values of solvation number indicate that the solutions are more compressible than the solvent. Many authors in literature report negative solvation number. ${ }^{24}$ These results indicate that strong solute-solvent interaction takes place in the solution. In general, one can consider two types of interactions between the component molecules such as physical and chemical interactions which are arising from dispersion forces or weak dipole-dipole interactions making a positive contribution and the chemical or specific interactions due to the charge transfer, hydrogen bond formation and other complex forming interaction results in negative values. ${ }^{25}$

\subsubsection{Micellar features}

The plots of $\mathrm{u}, \mathrm{Z}, \beta$ and $\mathrm{L}_{\mathrm{f}}$ against complex concentration (c) are characterised by an intersection of two straight lines corresponding to the CMC of the complex (Figures 1 to 4 ). It reveals that $\mathrm{CMC}$ is dependent on the composition of the solvent mixture. Like copper soaps derived from various edible oils in methanolbenzene mixture, copper soap-urea complex too also obeys Equation 10, showing a linear variation of ultrasonic velocity with complex concentration. The values of $\phi_{\mathrm{k}}$ increases with increasing complex concentration. The $\phi_{\mathrm{k}}$ values are negative for all urea complexes of copper soap. Literature survey reveals that negative value of $\phi_{\mathrm{k}}$ indicates electrostriction and hydrophobic interaction. ${ }^{26}$ The negative values of $\phi_{\mathrm{k}}$ are also attributed to the loss of structural compressibility of solute molecules. The plot of $\phi_{\mathrm{k}}$ vs. $\sqrt{\mathrm{c}}_{\mathrm{c}}$ is characterised by an intersection of two straight lines corresponding to the CMC of the complex (Figure 5). The $\phi_{\mathrm{k}}$ data has also been 
analysed in terms of Masson's equation. The parameters $\phi_{\mathrm{k} 2}^{0}$ and $\mathrm{S}_{\mathrm{k}}$ have been evaluated both below and above CMC. The order of this parameter is as follow:

$$
\phi_{\mathrm{k} 1}^{0}<\phi_{\mathrm{k} 2}^{0} \text { and } \mathrm{S}_{\mathrm{k} 1}>\mathrm{S}_{\mathrm{k} 2}
$$

From this order, it may be suggested that solute-solvent interaction is more pronounced in concentrate solution, i.e., above CMC as compared to below $\mathrm{CMC}$, this observation is in good agreement with our apparent molar volume $\phi_{\mathrm{v}}$ interpretations for complexes.

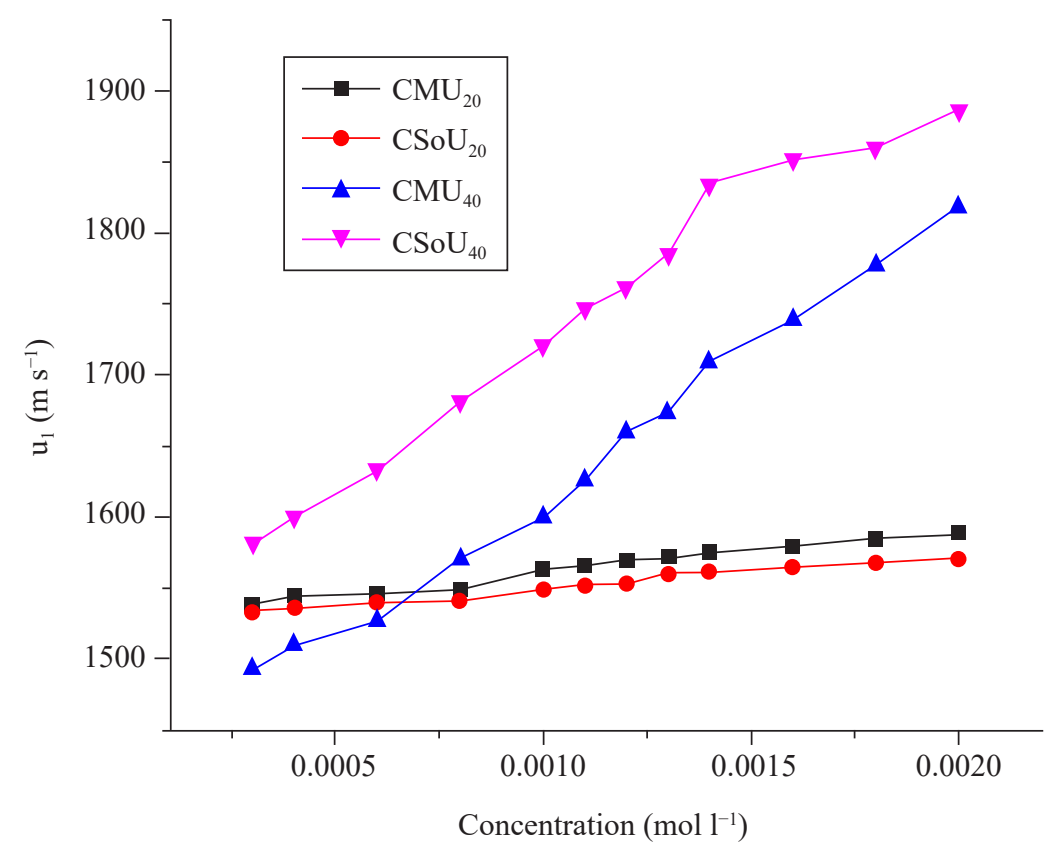

Figure 1: Plots of u vs. c of copper soap urea complex derived from mustard and soybean oil in $20 \%$ and $40 \%$ methanol-benzene mixture. 


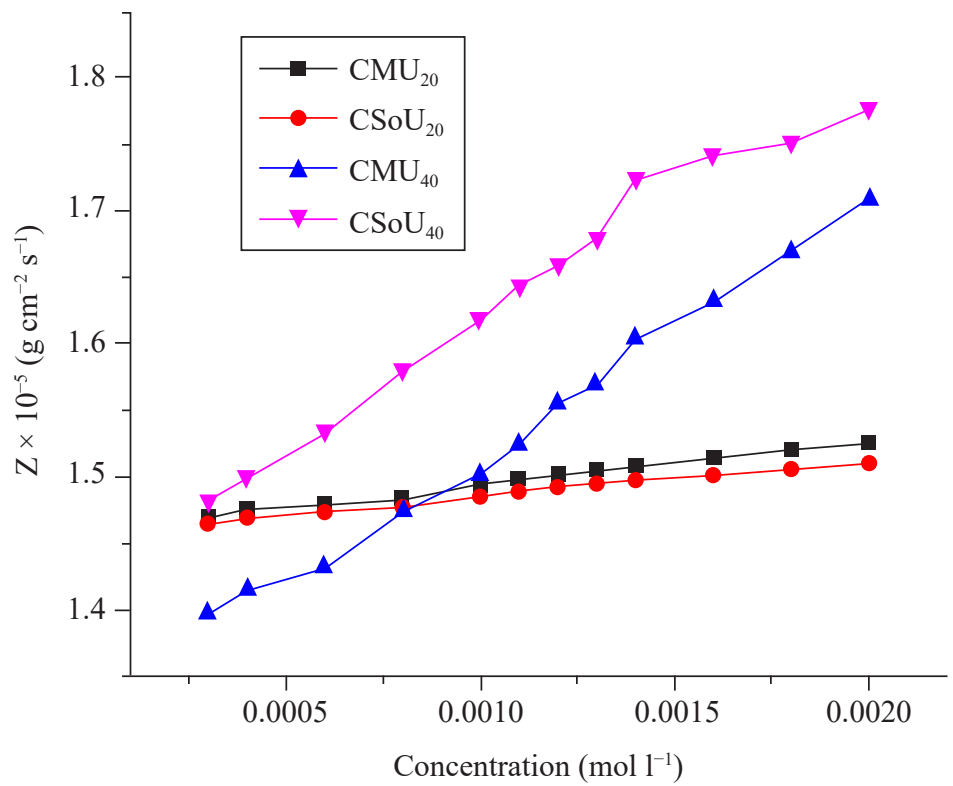

Figure 2: Plots of $Z$ vs. c of copper soap urea complex derived from mustard and soybean in $20 \%$ and $40 \%$ methanol benzene mixture.

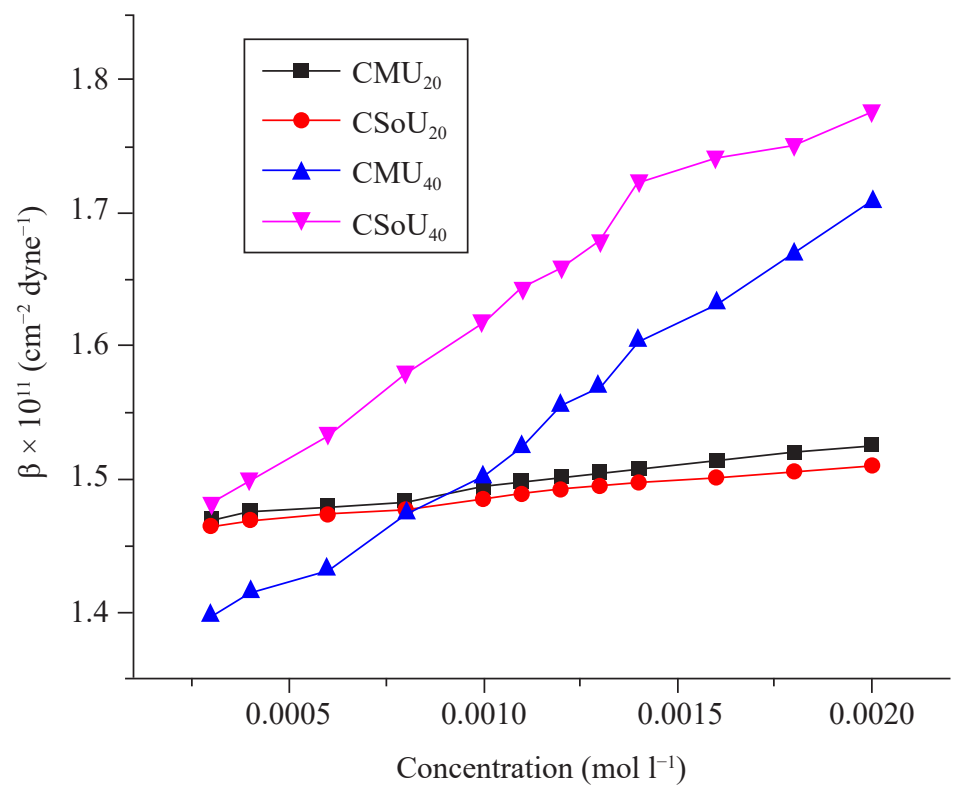

Figure 3: Plots of $\beta_{\mathrm{ad}}$ vs. c of copper soap urea complex derived from mustard and soybean in $20 \%$ and $40 \%$ methanol benzene mixture. 


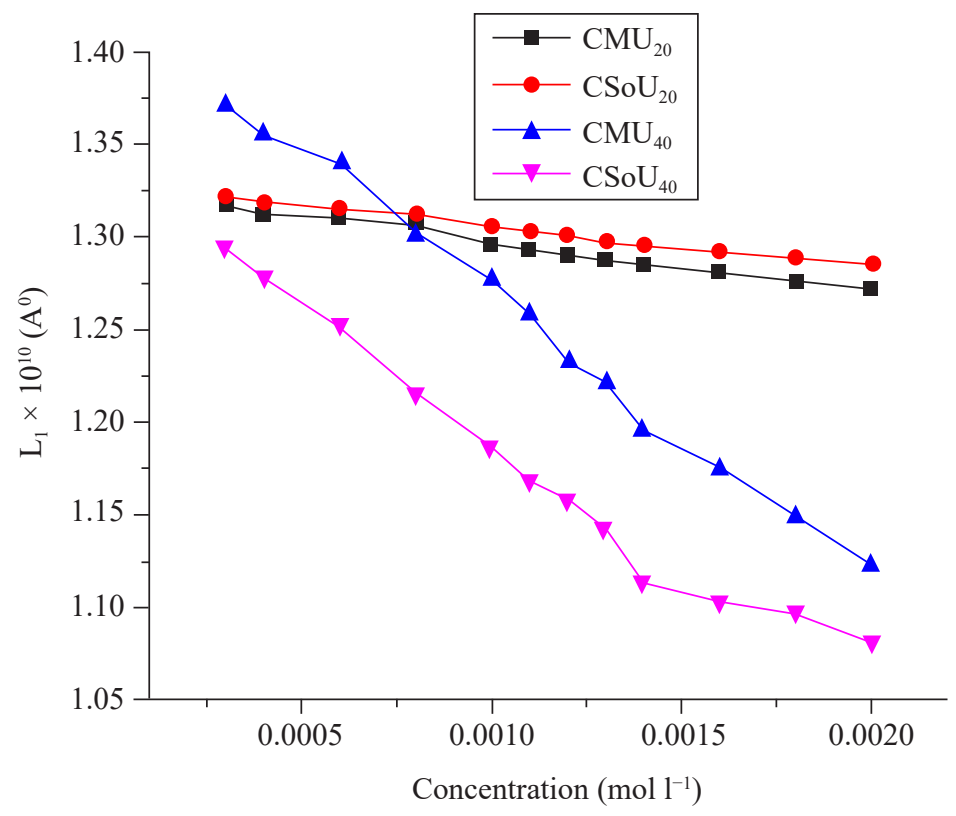

Figure 4: Plots of $\mathrm{L}_{\mathrm{f}}$ vs. $\mathrm{c}$ of copper soap urea complex derived from mustard and soybean oil in $20 \%$ and $40 \%$ methanol benzene mixture.

The CMC value follows the order:

$$
\mathrm{CSoU}>\mathrm{CMU}
$$

The results are in agreement with the fact that there is decrease in the CMC value with the increase in average molecular weight of the complex.

\subsection{Copper Soap-urea Complex Derived from Mustard and Soybean Oils in 40\% Methanol-benzene Mixture}

\subsubsection{Acoustic parameters}

At the very outset it may be mentioned that the acoustic parameters and related equations pertaining to ultrasonic investigations for the previously-referred system are almost the same as have been enumerated for the $40 \%$ methanol-benzene mixture and are recorded in Tables 5 and 6 . The data clearly indicate that the values of $\mathrm{u}, \mathrm{Z}, \phi_{\mathrm{k}}, \mathrm{R}$ and $\mathrm{S}_{\mathrm{n}}$ increase whereas the value of $\beta$ and $\mathrm{L}_{\mathrm{f}}$ decreases consistently with increase in the complex concentration in varying compositions of methanolbenzene mixture. ${ }^{27,28}$ 
Table 5: Ultrasonic velocity and other acoustic parameter of copper urea complex derived from mustard oil in $40 \%$ methanol-benzene mixture $\left(\mathrm{CMU}_{40}\right)$.

\begin{tabular}{ccccccccc}
\hline $\begin{array}{c}\mathrm{c} \\
\left(\mathrm{mol} \mathrm{l}^{-1}\right)\end{array}$ & $\begin{array}{c}\rho \\
\left(\mathrm{g} \mathrm{dm}^{-3}\right)\end{array}$ & $\begin{array}{c}\mathrm{u} \\
\left(\mathrm{m} \mathrm{s}^{-1}\right)\end{array}$ & $\begin{array}{c}\mathrm{Z} \times 10^{-5} \\
\left(\mathrm{~g} \mathrm{~cm}^{-2} \mathrm{~s}^{-1}\right)\end{array}$ & $\begin{array}{c}\mathrm{b}_{\mathrm{ad}} \times 10^{11} \\
\left(\mathrm{~cm}^{2} \mathrm{dyn}^{-1}\right)\end{array}$ & $\mathrm{L}_{\mathrm{f} \times 10^{10}\left(\mathrm{~A}^{0}\right)}-\phi_{\mathrm{k}} \times 10^{8}$ & $\mathrm{R} \times 10^{-2}$ & $\mathrm{~S}_{\mathrm{n}} \times 10^{-3}$ \\
\hline 0.0003 & 0.9360 & 1492.5 & 1.3970 & 4.7959 & 1.3712 & 1936.8 & 28.787 & -64.180 \\
0.0004 & 0.9368 & 1509.4 & 1.4140 & 4.6852 & 1.3553 & 1739.9 & 28.873 & -46.008 \\
0.0006 & 0.9375 & 1526.7 & 1.4313 & 4.5763 & 1.3394 & 1346.7 & 28.966 & -29.290 \\
0.0008 & 0.9384 & 1570.5 & 1.4737 & 4.3207 & 1.3015 & 1334.9 & 29.217 & -19.562 \\
0.0010 & 0.9389 & 1600.0 & 1.5022 & 4.1605 & 1.2771 & 1230.1 & 29.388 & -14.449 \\
0.0011 & 0.9370 & 1626.0 & 1.5236 & 4.0366 & 1.2580 & 1220.6 & 29.609 & -12.357 \\
0.0012 & 0.9374 & 1659.8 & 1.5559 & 3.8725 & 1.2321 & 1257.2 & 29.803 & -10.303 \\
0.0013 & 0.9378 & 1673.6 & 1.5695 & 3.8068 & 1.2217 & 1212.4 & 29.875 & -9.127 \\
0.0014 & 0.9382 & 1709.4 & 1.6038 & 3.6477 & 1.1958 & 1240.8 & 30.076 & -7.624 \\
0.0016 & 0.9386 & 1739.1 & 1.6323 & 3.5225 & 1.1751 & 1164.8 & 30.242 & -6.086 \\
0.0018 & 0.9392 & 1777.8 & 1.6697 & 3.3689 & 1.1492 & 1122.2 & 30.450 & -4.769 \\
0.0020 & 0.9399 & 1818.2 & 1.7089 & 3.2184 & 1.1233 & 1086.8 & 30.661 & -3.727 \\
\hline
\end{tabular}

Table 6: Ultrasonic velocity and other acoustic parameter of copper urea complex derived from soybean oil in $40 \%$ methanol-benzene mixture $\left(\mathrm{CSoU}_{40}\right)$.

\begin{tabular}{ccccccccc}
\hline $\begin{array}{c}\mathrm{c} \\
\left(\mathrm{mol} \mathrm{1}^{-1}\right)\end{array}$ & $\begin{array}{c}\rho \\
\left(\mathrm{g} \mathrm{dm}^{-3}\right)\end{array}$ & $\begin{array}{c}\mathrm{u} \\
\left(\mathrm{m} \mathrm{s}^{-1}\right)\end{array}$ & $\begin{array}{c}\mathrm{Z} \times 10^{-5} \\
\left(\mathrm{~g} \mathrm{~cm}^{-2} \mathrm{~s}^{-1}\right)\end{array}$ & $\begin{array}{c}\mathrm{b}_{\mathrm{ad}} \times 10^{11} \\
\left(\mathrm{~cm}^{2} \mathrm{dyne}^{-1}\right)\end{array}$ & $\mathrm{L}_{\mathrm{f}} \times 10^{10}\left(\mathrm{~A}^{0}\right)$ & $-\phi_{\mathrm{k}} \times 10^{8}$ & $\mathrm{R} \times 10^{-2}$ & $\mathrm{~S}_{\mathrm{n}} \times 10^{-3}$ \\
\hline 0.0003 & 0.9370 & 1581.0 & 1.4814 & 4.2695 & 1.2938 & 3711.0 & 29.313 & -51.011 \\
0.0004 & 0.9376 & 1600.0 & 1.5002 & 4.1662 & 1.2780 & 3049.1 & 29.413 & -36.295 \\
0.0006 & 0.9387 & 1632.7 & 1.5326 & 3.9966 & 1.2517 & 2324.8 & 29.582 & -22.046 \\
0.0008 & 0.9396 & 1680.7 & 1.5792 & 3.7678 & 1.2154 & 2034.9 & 29.845 & -14.385 \\
0.0010 & 0.9405 & 1720.4 & 1.6181 & 3.5923 & 1.1867 & 1807.9 & 30.055 & -10.185 \\
0.0011 & 0.9409 & 1746.7 & 1.6435 & 3.4834 & 1.1686 & 1744.2 & 30.196 & -8.518 \\
0.0012 & 0.9415 & 1762.1 & 1.6590 & 3.4207 & 1.1580 & 1653.7 & 30.268 & -7.409 \\
0.0013 & 0.9405 & 1785.7 & 1.6795 & 3.3344 & 1.1433 & 1588.1 & 30.437 & -6.370 \\
0.0014 & 0.9394 & 1834.9 & 1.7237 & 3.1619 & 1.1134 & 1593.1 & 30.752 & -5.023 \\
0.0016 & 0.9405 & 1851.9 & 1.7417 & 3.1005 & 1.1025 & 1435.8 & 30.815 & -4.096 \\
0.0018 & 0.9412 & 1860.5 & 1.7511 & 3.0696 & 1.0970 & 1295.2 & 30.845 & -3.506 \\
0.0020 & 0.9418 & 1886.8 & 1.7770 & 2.9826 & 1.0813 & 1210.5 & 30.975 & -2.828 \\
\hline
\end{tabular}

\subsubsection{Micellar features}

The plots of acoustic parameters viz. $\mathrm{u}, \mathrm{Z}, \beta$ and $\mathrm{L}_{\mathrm{f}}$ against complex concentration are characterised by an intersection of two straight lines (Figures 1 to 4 ). Before $\mathrm{CMC}$, there is slight curvature and after $\mathrm{CMC}$, the value of acoustic parameters are 
in a linear manner which corresponds to the CMC of complex. It is obvious from Table 7 that the $\mathrm{CMC}$ is depending upon the composition of the solvent mixture. Like other copper soap and their urea complexes, these complex also obeys the equation $\mathrm{u}=\mathrm{u}_{0}+\mathrm{Gc}$. The values of $\mathrm{G}$ and $\mathrm{u}_{0}$ were evaluated from the $\mathrm{u}$ vs. $\mathrm{c}$ plots and are recorded in Table 8.

Table 7: CMC values of copper urea complexes derived from mustard and soybean oils in $20 \%$ and $40 \%$ methanol-benzene mixture.

\begin{tabular}{lllll}
\hline Plot & $\mathrm{CMU}_{20}$ & $\mathrm{CSoU}_{20}$ & $\mathrm{CMU}_{40}$ & $\mathrm{CSoU}_{40}$ \\
\hline u vs. c & 0.0010 & 0.0013 & 0.0011 & 0.0014 \\
Z vs. c & 0.0010 & 0.0013 & 0.0011 & 0.0014 \\
b $_{\text {ad }}$ vs. c & 0.0010 & 0.0013 & 0.0011 & 0.0014 \\
$\mathrm{~L}_{\text {f vs. c }}$ & 0.0010 & 0.0013 & 0.0011 & 0.0014 \\
$\mathrm{f}_{\mathrm{k}}$ vs. Öc & 0.0010 & 0.0013 & 0.0011 & 0.0014 \\
\hline
\end{tabular}

Table 8: Values of various constants obtained from Masson's equation for copper urea complexes derived from mustard and soybean oils in $20 \%$ and $40 \%$ methanolbenzene mixture.

\begin{tabular}{lcccccc}
\hline Name of the soap & $\mathrm{U} \mathrm{m} \mathrm{s}^{-1}$ & $\mathrm{G} \times 10^{-5}$ & $\mathrm{f}_{\mathrm{k} 1}^{0} \times 10^{8}$ & $\mathrm{f}_{\mathrm{k} 2}^{0} \times 10^{8}$ & $\mathrm{~S}_{\mathrm{k} 1} \times 10^{8}$ & $\mathrm{~S}_{\mathrm{k} 2} \times 10^{8}$ \\
\hline $\mathrm{CMU}_{20}$ & 1533.5 & 0.3838 & -39.0 & -23.8 & 1.8040 & 0.9004 \\
$\mathrm{CSoU}_{20}$ & 1526.5 & 0.4452 & -35.0 & -21.0 & 1.8807 & 0.8390 \\
$\mathrm{CMU}_{40}$ & 1435.0 & 0.2867 & -23.2 & -14.6 & 1.0355 & 0.1853 \\
$\mathrm{CSoU}_{40}$ & 1515.0 & 0.3639 & -31.2 & -30.2 & 1.3763 & 1.1106 \\
\hline
\end{tabular}

This obviously suggests that the behaviour of complex in benzene-dominated environment and methanol dominated environment is quite different as they occupy different position in palisade layers of soap and complex agglomerations. ${ }^{29,30}$

With regards to solvent mixture $\mathrm{u}, \mathrm{Z}$ and $\phi_{\mathrm{k}}$ increases with the predominance of methanol in $40 \%$ methanol-benzene solvent mixture whereas decreases with the predominance of benzene in $20 \%$ methanol-benzene mixture. The results are viceversa for $\beta$ and $L_{f}$ for copper soap-urea complex system (Tables 3 to 6 ). ${ }^{31,32}$

The values of CMC of ultrasonic parameters are higher in $40 \%$ methanol-benzene mixture as compared to $20 \%$ methanol-benzene mixture. With regards to chain length of the soap segment of the complex molecules, the CMC follows the order:

$$
\mathrm{CSoU}>\mathrm{CMU}
$$


The plots of apparent molar compressibility $\phi_{\mathrm{k}}$ against square root of complex concentration $\sqrt{c}$ are characterised by an intersection of two straight lines followed by convex curve (Figure 5) and after $\mathrm{CMC}$, the value of $\phi_{\mathrm{k}}$ increases near the CMC. The values of $\phi_{\mathrm{k}}^{\circ}$ are evaluated from the intercept of the plots of $\phi_{\mathrm{k}} \mathrm{vs}$. $\sqrt{\mathrm{c}}_{\mathrm{c}}$ and values of $S_{k}$ are calculated from the slope of these plots and are recorded in (Table 8).

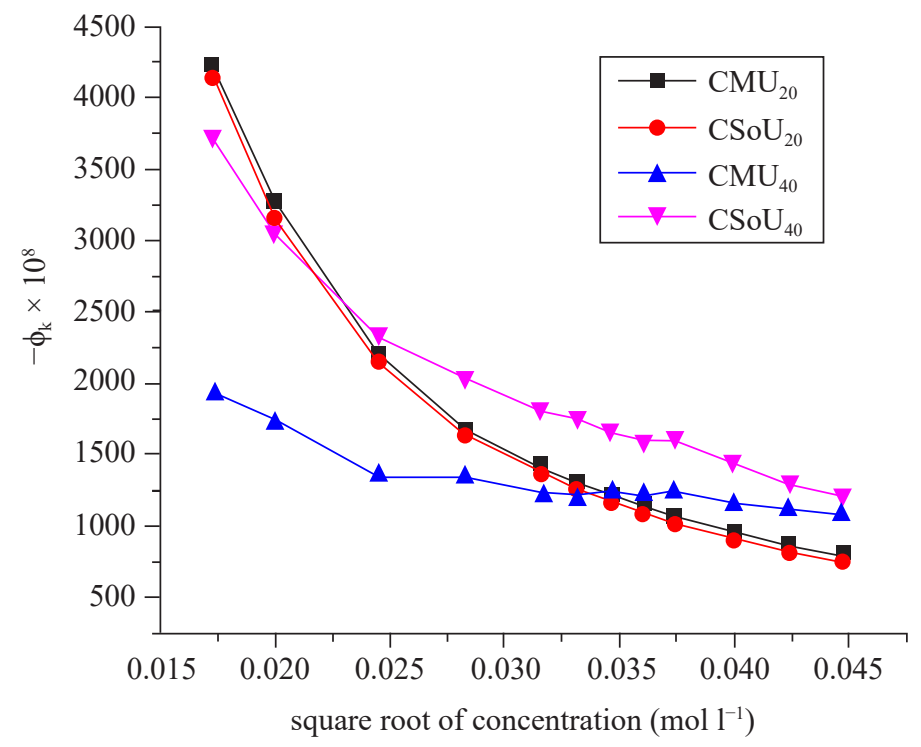

Figure 5: Plots of $\phi_{\mathrm{k}}$ vs. $\sqrt{\mathrm{c}}$ of copper soap urea complex derived from mustard and soybean in $20 \%$ and $40 \%$ methanol-benzene mixture.

The order of these parameters is as follows:

$$
\phi_{\mathrm{k} 1}^{0}>\phi_{\mathrm{k} 2}^{0} \text { and } \mathrm{S}_{\mathrm{k} 2}<\mathrm{S}_{\mathrm{k} 1}
$$

This suggests that solute-solvent interaction is more prominent in concentrate solution, i.e., above CMC as compared to below CMC. The value of $\phi_{\mathrm{k}}$ is higher in $40 \%$ methanol-benzene mixture as compared to $20 \%$ methanol-benzene mixture. The value of $\phi_{\mathrm{k}}$ is negative for all the copper urea complexes.

\section{CONCLUSION}

The nonlinear variation of apparent molar compressibility and other thermo acoustical parameters with molar concentration of copper soap-urea complex derived from mustard and soybean oils with $20 \%$ and $40 \%$ methanol-benzene mixture shows the intermolecular interaction occurs in constituent molecules. 
This provides useful information about the nature of intermolecular forces existing in the ternary mixture. The change in the behaviour below and above the CMC observed is attributed to the fact that aggregation of the soap-complex molecules is different in the ternary mixture of various concentrations which suggests that the solute-solvent interaction is more prominent in concentrate solution, i.e., above CMC as compared to below CMC. The value of $\phi_{\mathrm{k}}$ is higher in $40 \%$ methanolbenzene mixture as compared to $20 \%$ methanol-benzene mixture. The negative $\phi_{\mathrm{k}}$ values may be due to the loss of compressibility of solvent due to electrostatic forces in the vicinity of ions.

\section{ACKNOWLEDGEMENTS}

The authors pay their sincere gratitude to the University Grants Commission (UGC), New Delhi for financial support (UGCTRF no F. No: 25-159(12)/2003/ TRF/CRO), as well as S. D. Govt. College, Beawar, India and S. P. C. Govt. College Ajmer, India for providing necessary research facilities to accomplish this study.

\section{REFERENCES}

1. Rashmi, S. \& Arun, K. S. (2017). Natural edible oils: Comparative health aspects of sesame, coconut, mustard (rape seed) and groundnut (peanut) a biomedical approach. Biomed. J. Sci. Tech. Res., 1(5), https://doi.org/ 10.26717/BJSTR.2017.01.000441.

2. Tank, P. et al. (2017). A pharmaceutical approach and antifungal activities of copper soaps with their $\mathrm{N} \& \mathrm{~S}$ donor complexes derived from mustard and soyabean oils. Glob. J. Pharm. Sci., 3(4),1-6, https://doi.org/10.19080/ GJPPS.2017.03.555619.

3. Khan, S. et al. (2017). Antifungal activities of copper surfactants derived from neem (Azadirecta indica) and karanj (Pongamia pinnata) oils: A pharmaceutical application. Glob. J. Pharm. Sci., 3(4), 1-6.

4. Tank, P. et al. (2017). Thermal behaviour and kinetics of copper (II) soaps and complexes derived from mustard and soyabean oil. J. Anal. Pharm. Res., 4(2), 1-5, https://doi.org/10.15406/japlr.2017.04.00102.

5. Sharma, S. etal. (2017). Synthesis, characterization, and thermal degradation of $\mathrm{Cu}$ (II) surfactants for sustainable green chem. Asian J. Gr. Chem., 2(2), 129-140, https://doi.org/10.22631/ajgc.2017.95559.1015.

6. Sharma, A. K., Sharma, S. \& Sharma, R. (2017). Thermal degradation of $\mathrm{Cu}$ (II) metallic soaps and their characterizations. A pharmaceutical application. Chr. Pharm. Sci., 1(5), 312-319. 
7. Sharma, S. et al. (2017). Kinetic parameters and photo degradation studies of copper soap derived from soybean oil using $\mathrm{ZnO}$ as a photo catalyst in solid and solution phase. J. Inst. Chem. Ind., 89(4), 119-136.

8. Sivakumar, V. et al. (2007). Studies on the use of power ultrasound in solid liquid myrobal an extraction process. J. Clean. Prod., 15, 1813-1818, https://doi.org/10.1016/j.jclepro.2006.06.006.

9. Nath, G. (2012). Ultrasonic study of binary mixture of acetone with bromo benzene and chloro benzene at different frequencies. Chem. Sci. Trans., 1(3), 516-521, https://doi.org/10.7598/cst2012.171.

10. Singh, G. \& Banipal, T. S. (2008). Partial molar adiabatic compressibilities and viscosities of some amino acids in aqueous glycerol solutions at 298.15 K. Ind. J. Chem., 47A, 1355-1364.

11. Das, J. et al. (1999). Ultrasonic investigation in a polar-polar system, methyl-isobutyl ketone and aliphatic alcohols. J. Mol. Liq., 81, 163-179, https://doi.org/10.1016/S0167-7322(99)00065-3.

12. Ranganayakulu, S. V., Reddy, C. S. \& Reddy, D. L. (2005). Ultrasonic studies of the binary mixtures of ethyl acetate and cresols-application of Kosower and Dimroth treatments. Mat. Chem. Phys., 90, 213-216, https://doi.org/10.1016/j.matchemphys.2004.03.032.

13. Kanhekar, S. R., Pravina, P. \& Govind, K. B. (2010). Thermodynamic properties of electrolytes in aqueous solutions of glycine at different temperatures. Ind. J. Pure Appl. Phys., 48, 95-99.

14. Fort, R. J. \& Moore, W. R. (1965). Adiabatic compressibilities of binary liquid mixtures. Trans. Far. Soc., 61, 2102-2111, https://doi.org/10.1039/ TF9656102102.

15. Chauhan, M. (1992). Ultrasonic measurements on non-aqueous solutions of samarium soaps. J. Chem. Eng. Data, 37(4), 446-450, https://doi. org/10.1021/je00008a016.

16. Syal, V. K., Chauhan, S. \& Gautam, R. (1998). Ultrasonic velocity measurements of carbohydrates in binarymixtures of DMSO $+\mathrm{H} 2 \mathrm{O}$ at $25^{\circ} \mathrm{C}$. Ultrason., 36, 619-621, https://doi.org/10.1016/S0041-624X(97)80888-8.

17. Ali, A., Hyder, S. \& Nain, A. K. (1999). Intermolecular and ion solvent interaction of sodium iodide and potassium iodide in dimethyl formamide + I-propanol mixtures at 303K. J. Pure Appl. Ultrason., 21, 127-131.

18. Punitha, S. \& Uvarani, R. (2014). Physico-chemical studies on some saccharides in aqueous cellulose solutions at different temperaturesacoustical and FTIR analysis. J. Saudi Chem. Soc., 18, 657-665, https://doi.org/10.1016/j.jscs.2014.01.008. 
19. Kagathara, V. M., Sanariya, M. R. \& Parsania, P. H. (2000). Sound velocity and molecular interaction studies on chloroepoxy resins solutions at $30^{\circ}$ C. Eur. Polym. J., 36, 2371-2374, https://doi.org/10.1016/S00143057(00)00006-9.

20. Dash, A. K. \& Paikaray, R. (2013). Acoustical study on ternary mixture of dimethyl acetamide (DMAC) in diethyl ether and isobutyl methyl ketone at different frequencies. Phys. Chem. Liq., 51(6), 749-763, https://doi.org/ 10.1080/00319104.2013.795860.

21. Saxena, M., Sharma, R. \& Sharma, A. K. (2017). Micellarfeatures of Cu (II) surfactants derived from edible oils. Berlin: Lambert Academic Publishing.

22. Sharma, A. K., Singh, N. \& Saxena, M. (2017). Synthesis, spectroscopic and biocidal activity of $\mathrm{Cu}$ (II) sesame complex. Berlin: Lambert Academic Publishing.

23. Singh, S. \& Bahadur, I. (2014). Density and speed of sound of 1-ethyl-3methylimidazolium ethylsulphate with acetic or propionic acid at different temperatures. J. Mol. Liq., 199, 518-523, https://doi.org/10.1016/j.molliq. 2014.09.055.

24. Jahagirdar, B. V. et al. (2000). Studies in partial molar volumes, partial molar compressibilities and viscosity B-coefficients of caffeine in water at four temperatures. J. Mol. Liq., 85, 361-373, https://doi.org/10.1016/ S0167-7322(00)89019-4.

25. Naik, A. B. \& Narwade, M. L. (2014). Molecular interactions in substituted pyrimidines-acetonitrile solutions at 298.15-318.15 K. Russian J. Phys. Chem. A, 88(1), 37-41, https://doi.org/10.1134/S0036024414010324.

26. Jahagirdar, B. V. et al. (2000). Studies in acoustic properties, partial molar volumes, viscosity, B-coefficients of lithium chloride in aqueous medium at five temperatures. Ind. J. Pure Appl. Phys., 38, 645-650.

27. Khan, S., Sharma, R. \& Sharma, A. K. (2017). Ultrasonic studies of Cu (II) soap derived from seed oil of Pongamia pinnata (karanj), in non-aqueous binary and ternary systems at 298.15K. Mal. J. Chem., 19(2), 99-110.

28. Sharma, A. K., Saxena, M. \& Sharma, R. (2017). Ultrasonic studies of Cu (II) soaps derived from mustard and soybean oils. J. Pure Appl. Ultrason., 39(3), 92-99.

29. Tank, P., Sharma, R. \& Sharma, A. K. (2017). Studies of Ultrasonic and acoustic parameters of complexes derived from copper (II) surfactant of mustard oil with $\mathrm{N}$ and $\mathrm{S}$ atoms containing ligands in non-aqueous media (benzene) at 303.15 K. J. Acc. Soc. Ind., 44(2), 87-99.

30. Bhutra, R., Sharma, R. \& Sharma, A. K. (2017). Viscometric and CMC studies of $\mathrm{Cu}$ (II) surfactants derived from untreated and treated groundnut and mustard oils in non-aqueous solvent at 298.15 K. J. Inst. Chem. Ind., $90,29-47$. 
31. Sharma, A. K., Saxena, M. \& Sharma, R. (2018). Ultrasonic studies of Cu (II) soaps derived from groundnut and sesame oils. Tens. Surf. Det., 55(2), 127-134, https://doi.org/10.3139/113.110544.

32. Sharma, A. K., Saxena, M. \& Sharma, R. (2017). Acoustic studies of copper soap- urea complexes derived from groundnut and sesame oils. J. Phys. Stud., 21(4), 4601-4606. 